\title{
ENHANCED PROTOCOL FOR WIRELESS CONTENT-CENTRIC NETWORK
}

\author{
Chan-Min Park ${ }^{1}$, Rana Asif Rehman ${ }^{2}$, Tran Dinh Hieu², Byung-Seo Kim ${ }^{3}$ \\ ${ }^{1}$ Graduate School of Smart City Science Management, \\ Hongik University, Sejong, Republic of Korea \\ walkinpcmegmail.com \\ ${ }^{2}$ Dept. of Electronics and Computer Engineering, \\ Hongik University, Sejong, Republic of Korea \\ asifrehman7@gmail.com, trandinhhieu1989@gmail.com \\ ${ }^{3}$ Dept. of Computer and Information Communication Engineering, \\ Hongik University, Sejong, Republic of Korea \\ jsnbs@hongik.ac.kr
}

\begin{abstract}
Recently, Content-Centric Networking (CCN) was introduced and is expected as a new concept of future internet architecture. Even though CCN is initially studied for wired networks, recently, it is also studied for wireless environment. In this paper, we discuss improvement method for efficient content flooding over wireless CCNs. The proposed scheme of this paper use MAC Address of nodes when Interest and Data Packet are forwarded in order to limit the area of flooding of packets. The proposed protocol not only reduces the spread of Data packets, but also offers priority of forwarding to nodes of shortest path. As a consequence, it reduce content download time which is proved by extensive simulations.
\end{abstract}

\section{KEYWORDS}

Network Protocols, CCN, Future Internet, Protocol, Wireless.

\section{INTRODUCTION}

As mobile communication devices such as smartphones are widely used and rapidly spread out, so that peoples are easily connected through Internet, which is so-called Internet-of-People (IoP). Furthermore, such IoP not only increases tremendous of individuals data traffics, but also requests lots of huge contents such as video and music from content servers. Even though current Internet architecture provides efficiently end-to-end or host-to-host communications, it has been troubled to deal with user's mobility environment and services of aforementioned massive contents provided to unspecified number of users. Particularly, due to popularity of video streaming services such as TED and YouTube, more video-like content traffics are flooded over the networks. In such video streaming services, many users request even same content in a different time to same content server which might be located far from their locations. For example, let's assume that there is content server in U.S. and there are 10 people in Korea to

Jan Zizka et al. (Eds) : CCSEIT, AIAP, DMDB, MoWiN, CoSIT, CRIS, SIGL, ICBB, CNSA-2016

pp. 175-183, 2016. (C) CS \& IT-CSCP 2016

DOI : $10.5121 /$ csit.2016.60614 
request the content in a different time and different area. In this case, the content server will send same content 10 times from U.S. to Korea. This is very inefficient way to provide content. In order efficiently to request and provide the content itself, Content Centric Network (CCN) concept has been proposed in year 2009 by Van Jacobson [1]. In CCN, when the first content is delivered, we allow some server or router in Korea to store the content. Therefore, the second user's request on the same content can be provided from the nearby server or router, not far from server in U.S. Furthermore, CCN proposes to use a content name to request/distribute content instead of using IP address. That is, while current Internet focuses on "where to get" using IP address, $\mathrm{CCN}$ focuses on "what to get" using content name itself.

Even though initial studies for $\mathrm{CCN}$ have been conducted on the wired networks, recent studies moves to CCN-based wireless networks and is actively being conducted [2]-[10] because as mentioned earlier, people enjoys contents through smart handheld devices while they are moving. However, the research on wireless CCN is still early-state and there are many issues to be resolved. One of issue is long delay to download contents because of broadcasting and floodingbased transmissions adopted by wireless CCN. In this paper, we focus on how to reduce flooded traffics in wireless $\mathrm{CCN}$ in order to reduce download time of contents. To resolve the issue, we propose a novel protocol to reduce the number of content request messages, named Interest packet, flooded over the networks. As a result, the proposed protocol reduces collisions and wasted time in wireless $\mathrm{CCN}$.

In Section 2, the fundamentals of $\mathrm{CCN}$ and some prior arts related with wireless CCNs are introduced. In Section 3, after motivation of this research is introduced, the proposed protocol is described. In Section 4, the proposed protocol is evaluated in terms of the content download time and conclusions are made in Section 5.

\section{PRIOR ART}

$\mathrm{CCN}$ is composed of three devices and three packets [1]. Three devices are consumer, provider, and intermediate nodes, and three packets are Interest, Data, and Announcement packets. Each device consists of three components: Content Store (CS), Pending Interest Table (PIT), and Forwarding Information Base (FIB). A consumer requests a content by sending an Interest packet and the Interest packet is flooded over a network by all intermediate nodes. Once receiving the Interest packet, a node to check its CS if it has the content. If it has, it sends Data packets in the manner of broadcasting and it becomes a provider. If it doesn't, it records the information of Interest packet in its PIT and broadcasts the Interest packet to Networks. Therefore, any node that has request content can be a provider which is a content server in terms of conventional IP-based networks. When receiving a Data packet, then it checks PIT to ensure any node requested the content before. If yes, it broadcasts the Data packet. Otherwise, it discards the packet. In CCN, a provider can broadcast announcement packet to let nodes in network know specific content that the node has in CS.

After the concept of $\mathrm{CCN}$ was proposed, studies on wireless $\mathrm{CCN}$ have been actively performed [2]-[10]. Enhanced-Content-centric multiHop wireless NETwork (E-CHANET) is designed for wireless networks adopting CCN concept [2]. In E-CHANET, Announcement packet and FIB component are removed from the system. Instead, to cope with erroneous wireless channel, it uses Interest-Data-based two-way handshake performed between consumer and provider. Therefore, every Data packet transmission is requested by one Interest packet. If there is a lost 
Data packet, same Interest packet is retransmitted. Calculating wait-time for receiving the requested Data packet and handoff method between different providers are also designed. The CCN communication method is also applied to vehicular networks [4]. CarSpeak enables a car to query and access sensory information captured by other cars in a manner similar to a way to request a content in content-centric approach. Authors in [4] try to solve packet loss problem caused by node mobility in CCN-based MANETs. In [4], both of broadcast and unicast are used for the transmissions of Interest packets, and additional function is added which is to validate if the next hop node is available when a node receives Interest or Data packet. Flooding issue in $\mathrm{CCN}$ has been studied in [5][6]. In [5], the shortest hop count is found by flooding Interest packets and then Data packet is flooded back to consumer within the hop count. In [6], the authors proposed a new scheme called Neighborhood-Aware Interest Forwarding (NAIF) for NDN-based mobile ad hoc networks. This scheme basically reduces the flooding overhead in the network. In which, the intermediate node locally decide to propagate or drop the received Interest packet based on the forwarding rate. Authors in [7] propose a scheme for multihop based wireless CCN. Two additional packets, called EFS-ACK and EFS are also utilized in addition to the Interest and Data packets. In [8], the authors present an energy aware forwarding scheme for multihop wireless ad hoc networks. In which, the packets are forwarded based on node's residual energy. Authors in [9] propose forwarding strategies (i.e. BF, PAF) for wireless CCN ad hoc networks. These schemes are deeply analyze and well evaluated. Furthermore, the authors also highlight the advantages and shortcoming of both approaches. Kim et al., in [10], present a novel scheme, named AIRDrop in which communication is based on unicast manner. Proposed AIRDrop scheme also takes into account the extra tables and buffers during its communication operations.

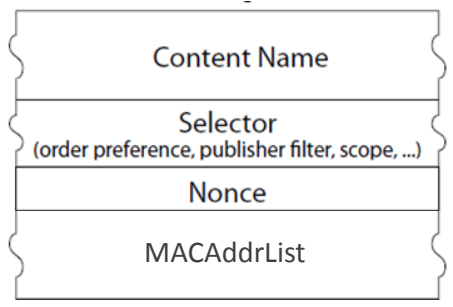

(a)

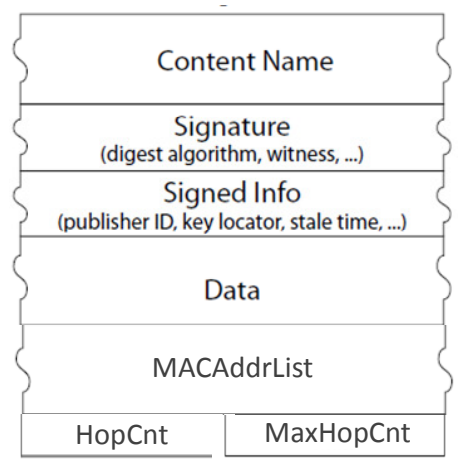

(b)

Figure. 1. Revised packet formats for the proposed protocol: (a) Interest packet and (b) Data packet

\section{PROPOSED PROTOCOL}

\subsection{Motivation}

Although previously proposed protocols for $\mathrm{CCN}$-based wireless networks provide advantages for delivering contents, their performance is still struggling in terms of content download time. One of the reasons is inefficient Data packet forwarding mechanism. In wireless environments, using broadcasting manner, the direction of packet flows cannot be controlled, so that too much flooding causes delays to download contents. Since many wireless routing protocols uses unicasting and IP addresses, so that packet's flow can be controlled using IP addresses. However, because wireless CCNs do not use IP address and use broadcasting address for MAC protocol, it 
is hard to restrict flooding area and control the content flows. On the other hand, totally removing flooding in wireless CCNs also removes advantage of CCNs' nature which is to spread contents over the networks for possible upcoming content request. Therefore, the objective of this paper is to propose a protocol for wireless CCNs which not only limits flooding of Data packets in a certain range over the networks, but also speeds up content download time using a shortest path from a provider to a consumer.

In the proposed protocol, the way to forward Interest packet is same as that of E-CHANET. However, whenever nodes forwards the packet, they add their MAC addresses in the packet. When the list of MAC addresses of the Interest packet arriving firstly at a provider, it will be used as the shortest path for Data packet's forwarding. Furthermore, since nodes in the list are allowed to have priorities to access the channel, Data packets can be arrived at a consumer faster comparing to the conventional wireless CCNs. Unlike common unicasting routing protocols used in wireless ad-hoc networks, the Data packets is not forwarded only by the nodes in the list. The packet is also forwarded in some area of the nodes in the list. That is, the Data packets are flooded in a restricted area around the shortest path. Therefore, it achieves not only a better content download time, but also flooding of Data packets.

\subsection{New Frames}

In the proposed protocol, Interest and Data packets' formats of E-CHANET are modified as shown in Fig.1. As shown in Fig. 1(a), for the Interest packet of the proposed protocol, one field, called MACAddrList, is added at the end of the packet. This field includes MAC addresses of intermediate nodes between a consumer and a provider. When a node receives an Interest packet and decides to forward it, it adds its MAC address in the field. Therefore, MAC addresses are accumulated in the field as the Interest packet is forwarded to a Provider, so that the size of the field is varied according to the number of nodes forwarding the Interest packet before it arrives at a provider. This filed is also used for Data packet as shown in Fig. 1(b). The list of addresses in the field of Interest packet is copied to the field of Data packet by a provider before the Data packet is transmitted. While a node adds its MAC address into MACAddrList field when receiving an Interest packet, it removes its MAC address from the field of Data packet when it receives the data packet and its address is in the field. MaxHopCnt is the number of MAC addresses indicating how many nodes the Interest packet have come through from a consumer to the provider. HopCnt field in Data packet indicates how many hops the Data packet can be forwarded. That is, this limits forwarding Data packet unnecessary further. When a node receives a Data packet, it recalculates HopCnt which is explained in detail in Section 3.3. HopCnt is differently calculated depend on whether or not the node itself is in MACAddrList.

\subsection{Operation of the proposed protocol}

In this section, operations of the protocol are explained in two parts: cases when a node receives Interest packet and Data packet.

\section{Process when a node receives an Interest packet.}

The procedure of the proposed protocol when a node receiving an Interest packet is shown in Fig. 2. 


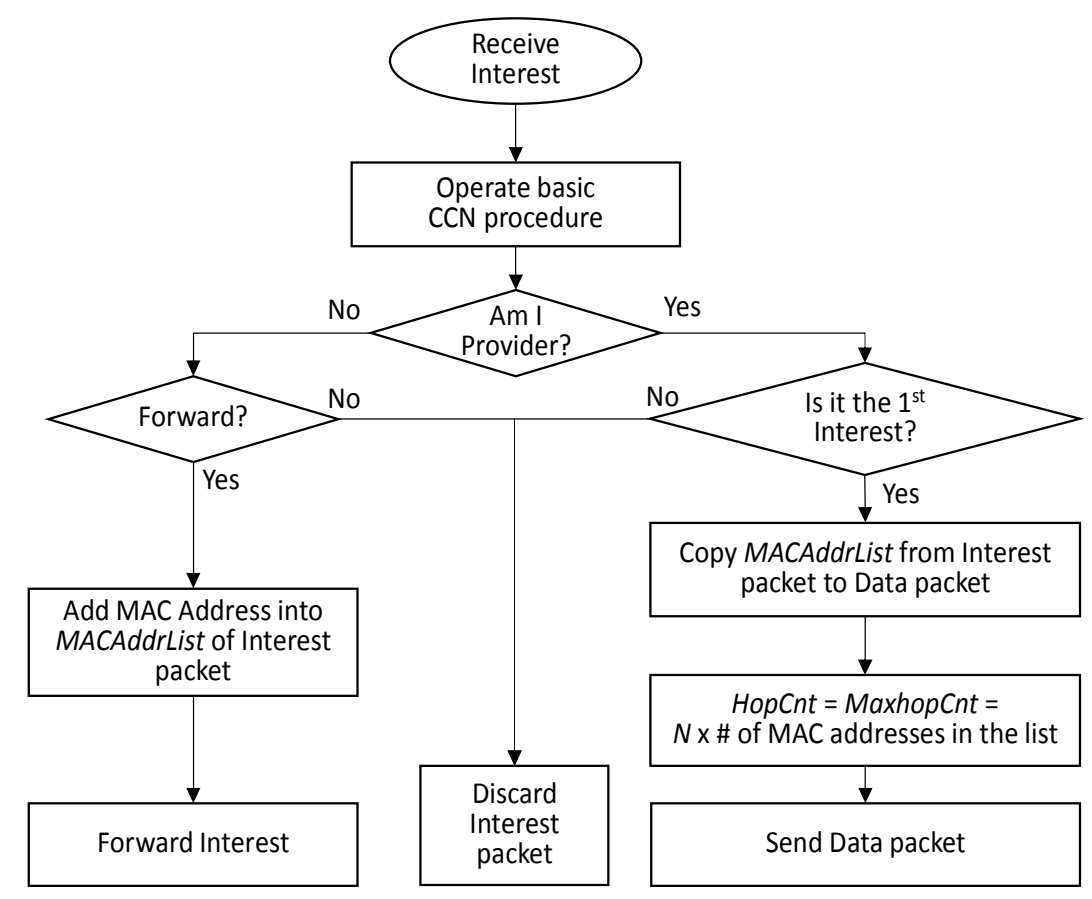

Figure. 2. Procedure of the proposed protocol when a node receives Interest packet

When a node receives an Interest packet, it performs the process defined from conventional wireless CCN such as E-CHANET. That is, it checks CS and PIT and decides whether or not to forward the packet. If the node acts as a relay node and the received Interest packet needs to be forwarded, it add the last 24bits of its MAC address to MACAddrList field in the received Interest packet and forwards the packet to the neighbours. If the packet is already received before, the node discards the packet.

If the node becomes a provider (that means it has the requested contents in its CS), it checks if it already received an Interest packet requesting same content and nonce number. If it did, it discards the packet. Otherwise, it records the first packet requesting the content with the nonce is received, and then at first, it copies the list in MACAddrList field in the Interest packet to MACAddrList field in Data packet to be sent. Secondly, it set HopCnt field to $\mathrm{N}$ times the number of MAC addresses in the field. Finally, it sends the Data packet to Consumer.

In the process, when a provider receives an Interest packet, it sets MaxHopCnt to $\mathrm{N} x$ the number of MAC addresses in MACAddrList of the Interest packet.

\section{Process when a node receives a Data packet.}

When a node receives Data packet, it processes conventional CCN process. Then it checks if it need to forward the received Data packet. If it does not need to forward the packet, it discards the packet. If it need to forward it, but HopCnt is 0 , it discards the packet. Otherwise, the node checks if there is its own MAC address in the MACAddrList of the Data packet. If there is, HopCnt of the Data packet is set to MaxHopCnt and the node's own MAC address is removed from MACAddrList of the packet. In addition, after choosing random number between 0 and $W_{s}$, 
it sets its deferring time, DeferT, to the random number x slot time, SlotT. After waiting DeferT, it forwards the Data packet to neighbours. If there isn't its own MAC address in the MACAddrList of the Data packet, the node checks if HopCnt of the packet equals to 1 . If it is, HopCnt is set to 0. Otherwise, HopCnt is set to the integer that is not higher than root of HopCnt of the packet. After choosing random number between 0 and $W_{n}$, it sets its DeferT, to the random number x Slot T. After waiting DeferT, the packet is forwarded to the neighbours.

The proposed protocol provides two unique characteristics to efficiently forward Data packets. The first characteristic is to limit the Data packet's flooding by exponentially reducing HopCnt as explained in Step 4-2a. If a node receiving the Data packet coming from the non-shortest path, the Data packet is forwarded within number of hops. If it is coming from the shortest path, its Data packet is flooded as many hops as MaxHopCnt as explained in Step 4-1a. Therefore, it maintains an advantage of conventional CCN's flooding as well as prevents from unnecessary packet flooding. The second characteristic is to give transmission priority to nodes on the shortest path as explained in Step 4-1c and 4-2c. In conventional wireless CCNs, since all data packets are broadcasted in MAC payer, there is no retransmissions and backoff time increase. Because of this, it causes lots of collisions due to concurrent transmissions. Therefore, to resolve concurrent transmissions, CCN layer provides Deferred Time which a node waits random time before transmission. In this case, to give better transmission opportunity to the nodes along with the shortest path, the proposed protocol allows them to choose random number from the smaller range of numbers $(0 \sim \mathrm{Ws})$ than other nodes. The other nodes choose the random number from the wider range of numbers $(0 \sim \mathrm{Wn})$. As a result, nodes on the shortest path have the higher transmission opportunities than others.

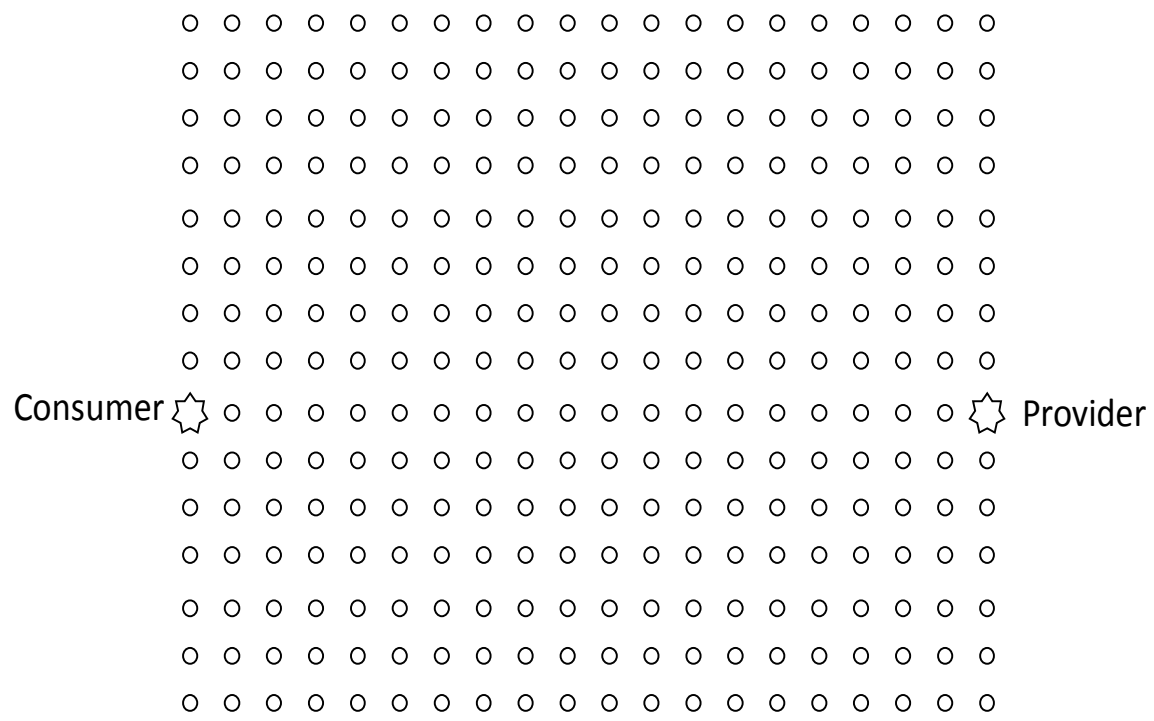

Figure. 3. Network Topology for the simulations 


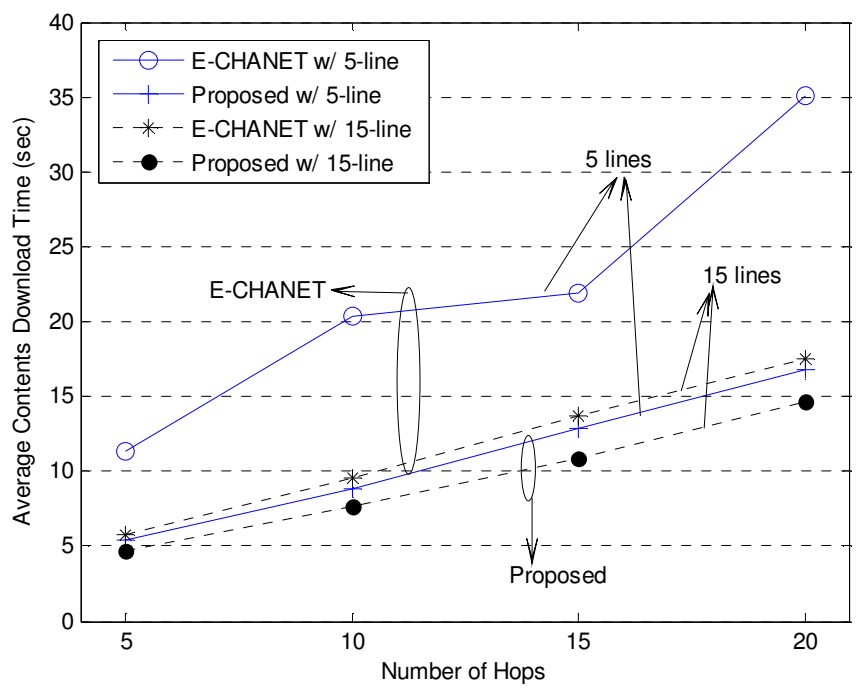

Figure. 4. Average content download time as a function of the distance between a consumer-provider pair

\section{Performance Evaluations}

In this section, the proposed protocol is comparatively evaluated with E-CHANET-based protocol. For evaluation studies, we have used ndnSIM [10] software module that is based on Network Simulator-3 (NS-3) version 3.16. In the simulations, one content consists of 100 packets and payload length of a data packet is set to 1200 bytes. Physical layer transmission rate is set to $6 \mathrm{Mbps}$ and 5GHz-carrier frequency-based IEEE802.11a standard is adopted. Medium access control protocol is based on IEEE802.11e standard. For the channel model, log normal path loss model with path loss exponent 3 is used. The simulations results are collected from 100 times simulations and one simulation completed when a consumer receives all 100 chunks packets from a provider.

The performances are evaluated as functions of network sizes by varying distances of a consumer-provider pair and the number of lines of Y-axis in grid topology. The topology for the simulations are shown in Fig. 3. Fixing a consumer's location, performances are evaluated varying distances to a provider. The distances are 5, 10, 15, 20 hops. In addition to varying distances between the consumer and the provider, the grid sizes are varied as like 5 and 15 lines. 1 line means there is only one path between the consumer and the provider. That is, the number of hop indicates the number of relay nodes in $\mathrm{x}$-axis over grid topology while the number of lines indicates the number of relay nodes in y-axis in the topology. Therefore, we evaluates the performances over 8 sizes of networks (4 types of hops and 2 types of lines).

Fig. 4 shows the average content download time as varying the distance between a consumer and provider when the numbers of lines are 5 and 15. As mentioned before, the distance is represented using the number of hops between the consumer-provider pair and the distance is varied from 5 to 20 hops. As shown in the figure, the proposed protocol reduces the download time from $16 \%$ to $52 \%$ comparing to E-CHANET protocol. From the figure, as the distance increases, the reduction of download time is larger. The reason is because E-CHANET protocol makes more packets flooded over the networks as the distance increase. Since higher traffics over 
the networks causes higher download time because of packet losses due to collisions and longer backoff time in MAC layer. It is noticed that there is exceptionally long download time of ECHANET protocol in 5 lines in Fig. 4. This can be explained from Fig. 5 showing the average number of retries. As Fig. 5 shows, E-CHANET protocol has the highest number of retries. Since the forwarding path becomes narrower, the advantage of E-CHANET protocol's flooding-based Data packet forwarding decreases. Even though flooding-based packet forwarding method cause lots of collisions because too many nodes are participating in transmissions, the packet can eventually delivered to the consumer through many alternative paths because it is flooded. However, in the narrower network like 5 lines, the number of alternative paths are limited, so that the advantage of flooding decreases. Therefore, the number of retransmissions increases which leads the download time's increase.

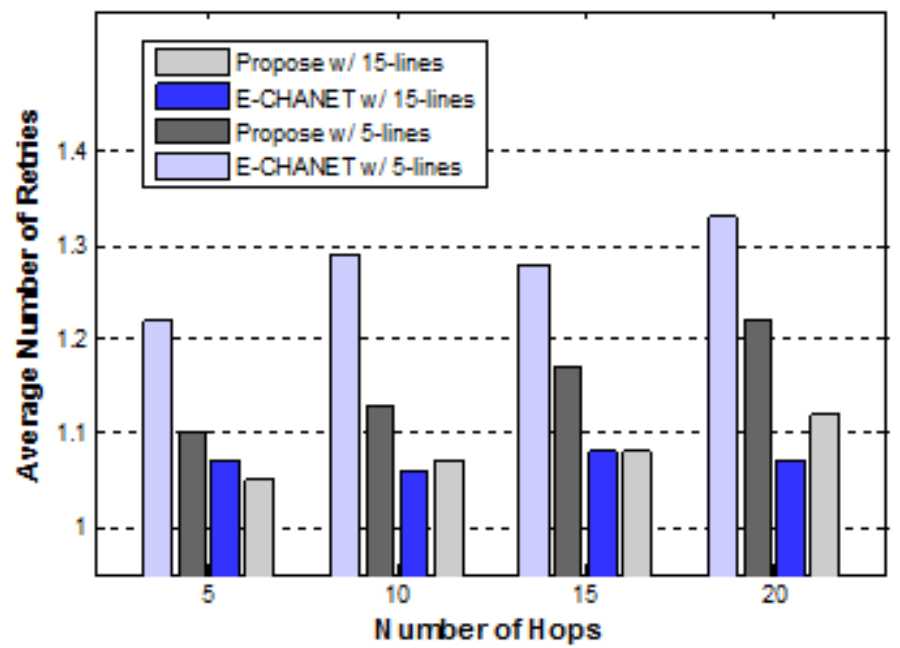

Figure. 5. Average number of retries as a function of the distance between a consumer-provider pair

\section{CONCLUSIONS}

In this paper, a novel protocol for wireless CCNs is proposed. Even though the protocol uses broadcasting and flooding-based Data packet forwarding, by restricting flooding area and giving transmission priority to a nodes on the shortest path, the protocol resolves the long content download time issue. The proposed protocol is evaluated throughout extensive simulations and it is proved that the objective of the proposed protocol successfully achieved by reducing a content download time up to $50 \%$ comparing to the conventional protocol.

\section{ACKNOWLEDGEMENTS}

This research was supported in parts by Basic Science Research Program through the National Research Foundation of Korea (NRF) funded by the Ministry of Education (2013R1A1A2005692) and in parts by the International Science and Business Belt Program through the Ministry of Science, ICT and Future Planning (2015K000270). 


\section{REFERENCES}

[1] V. Jacobson, D.K. Smetters, J.D. Thornton, M.F. Plass, N.H. Briggs, and R.L. Braynard, "Networking named content," Proc. Int. Conf. on Emerging Networking Experiments and Technologies, (CoNEXT’09), Rome, Italy, Dec. 2009. pp. 1-12.

[2] M. Amadeo, A. Molinaro, and G. Ruggeri, "E-CHANET: routing, forwarding and transport in information-centric multihop wireless networks," Computer Communications, vol. 36, no. 7, pp. 792803, April 2013.

[3] M Amadeo, C Campolo, and A Molinaro, "Enhancing content-centric networking for vehicular environments," Computer Networks, vol. 57, no. 16, pp. 3222-3234, November. 2013.

[4] O. Adem, S. Kang, Y.B. Ko, "Packet Loss Avoidance in Content Centric Mobile Adhoc Netowrks," 15th International Conference on Advanced Communication Technology (ICACT'13), pp. 245-250, PyeongChang, Korea, January 2013.

[5] H. Han, M. Wu, Q. Hu, and N. Wang, "Best Route, Error Broadcast: A Content-Centric Forwarding Protocol for MANETs," IEEE 80th Vehicular Technology Conference (VTC Fall), pp. 14-17, Vancouver, Canada, September 2014.

[6] Y.-T. Yu, B.R. Dilmaghani, S. Calo, M.Y. Sanadidi, and M. Gerla, "Interest propagation in named data manets," in Proc. of IEEE International Conference on Computing, Networking, and Communications (ICNC'2013), pp. 1118-1122, San Diego, CA, January 2013.

[7] D. Kim and Y.-B. Ko, "A novel message broadcasting strategy for reliable content retrieval in multihop wireless content centric networks," In Proceedings of the 9th ACM International Conference on Ubiquitous Information Management and Communication, Bali, Indonesia, January 2015.

[8] R.A. Rehman and B.-S. Kim, "Energy aware forwarding in content centric based multihop wireless ad hoc networks," IEICE transactions on on Fundamentals of Electronics, Communications and Computer Sciences, vol. E98-A, no. 12, pp. 2738-2742, December 2015.

[9] M. Amadeo, C. Campolo, and A. Molinaro, "Forwarding strategies in named data wireless ad hoc networks: Design and evaluation," Journal of Network and Computer Applications, vol. 50, pp. 148158, April 2015.

[10] D. Kim, J.-H. Kim, C. Moon, J. Choi, and I. Yeom, "Efficient content delivery in mobile ad-hoc networks using ccn," Ad Hoc Networks, vol. 36, pp. 81-99, January 2016.

[11] A. Afanasyev, I. Moiseenko, and L. Zhang, "ndnSIM: NDN simulator for NS-3," Technical Report NDN-0005, NDN, October 2012. 